RAL Report

RAL-94-098

\title{
Dynamical Properties of Critical and Paramagnetic Spin Fluctuations in Anisotropic Heisenberg Magnets
}

S W Lovesey and E Balcar

September 1994 
DRAL is part of the Engineering and Physical

\section{Sciences Research Council}

The Engineering and Physical Sciences Research Council does not accept any responsibility for loss or damage arising from the use of information contained in any of its reports or in any communication about its tests or investigations 


\title{
Dynamical Properties of Critical and
}

\section{Paramagnetic Spin Fluctuations in Anisotropic Heisenberg Magnets}

\author{
S. W. Lovesey, DRAL, Rutherford Appleton Laboratory \\ Oxfordshire OX11 0QX, England, U.K. \\ E. Balcar, Atominstitut der. Österr. Universitäten, \\ A-1020 Wien, Austria
}

\begin{abstract}
Decay rates for long wavelength spin fluctuations in the standard $x-y$ model, and a second model described by the sum of the full Heisenberg exchange interaction and a single-site anisotropy are calculated on the basis of a coupled-mode theory in which the isothermal susceptibilities are consistent with corresponding spherical models (in consequence, quantum fluctuations are absent). Results for the decay rates are provided for two and three dimensional lattices, and ferromagnetic and antiferromagnetic exchange interactions. Their dependence on the wave vector, $q$, and the inverse correlation lengths are derived from integral equations that admit homogeneous functions as solutions. The decay rates may thus be expressed as $q^{d / 2} R_{1}\left(q / \kappa_{0}\right)$ and $q^{(d+2) / 2} R_{2}\left(q / \kappa, q / \kappa_{0}\right)$ where $R_{1}$ and $R_{2}$ are, respectively, scaling functions for the $x-y$ and ferromagnetic single-site anisotropy models, $d$ is the spatial dimension, and $\kappa\left(\kappa_{\mathrm{o}}\right)$ is the out-ofplane (in-plane) inverse correlation length. Analytical and numerical values of the scaling functions are presented for the limits of main physical interest.
\end{abstract}





\section{Introduction}

We report findings from calculations of the time dependence of spin fluctuations in two magnetic models that have in common lattice isotropy and spin space anisotropy: (I) the $x-y$ model, in which the usual Heisenberg exchange interaction between neighbouring spin variables, $\left\{\mathbf{S}_{a}\right\}$, is truncated to two of the three Cartesian components of the spins, usually labelled the $x$ and $y$ components, and (II) the full Heisenberg exchange interaction plus a single-site anisotropy of the form, $-B\left(S_{a}^{z}\right)^{2}$. The latter model is believed to furnish an appropriate description of a number of magnetic materials, e.g. the rutile antiferromagnets $\mathrm{MnF}_{2}$ and $\mathrm{FeF}_{2}$ (Collins 1989 , Cowley 1987). Early studies of critical spin fluctuations in model-II are reviewed by Kawasaki (1976), and a variant of it is included in the range of models studied by Bagnuls and JoukoffPiette (1975). The $x-y$ model dates back to 1956 and it is now a standard model in statistical mechanics; its static properties calculated for quantum spins (mainly spin-1/2) are reviewed by Betts (1974), Hohenberg and Halperin (1977), and Mattis (1985). The $x$ - $y$ model is relevant to the interpretation of properties of liquid helium and displacive transitions exhibited by ferroelectric materials, in addition to some anisotropic magnets. In this model, the order parameter, the total $x$-component of the spin, say, does not commute with the Hamiltonian. In fact, both models have this feature which has a profound effect on their dynamical properties, as we will see. The total $z$-component of the spin in the $x-y$ model and the model with a single-site anisotropy, on the other hand, is a constant of the motion. If the anisotropy parameter, $B$, is negative the anisotropy suppresses fluctuations in the $z$-components of the spins, and with an increasing magnitude of the anisotropy parameter the model approaches a planar system. While similar to the $x-y$ model, of course, the planar limit of model-II is a fundamentally different system since it has almost two spin degrees of freedom, whereas in model-I all three spin degrees of freedom fully participate in its static and dynamical properties. By way of an illustration of the differences in the properties of the two models, we anticipate the results for the dynamic critical exponent, $z$. For model-I (ferromagnetic II) we find $z=d / 2(1 / 2(d+2)$ ) where $d$ is the spatial dimension of the lattice. The different values of $z$ for the two models is due in the $x-y$ model to the absence of out-of-plane exchange interactions, so this component of the spin density mimics a perfect paramagnet with a Pauli susceptibility. 
Both spin models taken on a three dimensional lattice (3D) are believed to display longrange magnetic order below a critical temperature, $T_{\mathrm{c}}$. Previous work on their dynamical properties has largely focused on the nature of the critical fluctuations at, and in the immediate vicinity of, the continuous phase transition at $T_{\mathrm{c}}$. By contrast, for two dimensional lattices (2D) the models do not appear to support long-range magnetic order at a finite temperature; for a review of the static properties, see Landau (1993). This observation for the $x-y$ model is supported by the Mermin-Wagner (1966) theorem for models with a continuous spin symmetry. There is a subtlety with the quantum $x-y$ model on a $2 \mathrm{D}$ lattice, for there is some evidence that the static susceptibility, specific heat, etc., diverge at a finite temperature. An implication is that below the transition the spin autocorrelation function does not decay exponentially but rather as some power law. The nature of the phase transition is possibly akin to the one believed to occur in the pure planar model, where it is ascribed to a spontaneous generation of vertex (topological) excitations (Berezisnkii 1971, Kosterlitz and Thouless 1973). In conclusion, for the $2 \mathrm{D}$, quantum versions of the models under investigation our knowledge about the static correlations is by no means complete, to the extent that the ground state of the $x-y$ model is not known (for any spatial dimension).

Our models are defined to possess the static correlation functions of the appropriate spherical models. In consequence, our models do not contain quantum mechanical fluctuations. The spherical models in 3D support long-range correlations at a finite temperature, and in $2 \mathrm{D}$ at a finite temperature there is no long-range order and no discontinuities in macroscopic response functions. It is found that in $2 \mathrm{D}$ the spin space anisotropy increases in correlation length compared to its value in the isotropic Heisenberg model, i.e. as a result of the spin anisotropy the $2 \mathrm{D}$ models are that much closer to long-range magnetic order.

Our investigations of model-I, II have been conducted for 2D and 3D lattices with ferromagnetic (F) and antiferromagnetic (AF) exchange couplings (NB, for the $x-y$ model, and model-II with planar anisotropy the critical fluctuations do not depend on the nature of the exchange coupling). Calculations are based on the coupled-mode approximation for spin fluctuations. For a ferromagnetic coupling there are two coupled equations of motion, for the interacting in-plane $(x, y)$ and out-of-plane spin fluctuations, and for an antiferromagnetic coupling the number of equations is doubled because important fluctuations occur at the 
Brillouin zone centre and the wave vector that defines the incipient antiferromagnetic ordering. In the coupled-mode approximation, each equation of motion is an integral-differential equation that relates the spin response function to its memory function, which is a spatial convolution of products of spin response functions, see e.g. Lovesey (1986). To ascertain the nature of the dynamic spin fluctuations it is sufficient to study the decay rates for the relevant, long wavelength fluctuations (this is sometimes referred to as the use of a Markovian approximation). While this level of treatment of the equations of motion does not provide insight to possible deviations of power spectra from a Lorentzian shape, decay rates can be extracted from appropriate experimental data (Collins 1989, Cowley 1987). Hence, by calculating decay rates, which are functions of the wave vector and temperature, we characterize the nature of the relevant fluctuations and provide quantities which can be compared to experimental findings in the appropriate cases. The decay rates for both models are homogeneous functions. We provide analytical and numerical values for the scaling functions in the limit that are of main physical interest.

With regard to $2 \mathrm{D}$-systems, there is a dearth of magnetic materials which are really well described by either of our two models, in the manner of the very strong evidence that the AF isotropic Heisenberg model describes $\mathrm{RbMnF}_{3}$. However, appropriate materials might be discovered. For the moment, our findings for the two models on 2D-lattices are a contribution to the zeitgeist in the statistical mechanics of magnetic models. To the best of our knowledge, there is no prior work on the application of the coupled-mode theory to the models we have completely specified in terms of spherical models and the coupled-mode theory.

The model Hamiltonians are given in the next section, and the corresponding coupledmode equations, on which our calculations of the decay rates are based, are set out in $\$ 3$. Section 4 is given over to a detailed discussion of static spin correlation functions which complete the specification of the models. The static susceptibilities are derived from the coupled-mode equations via the f-sum rule, and they are consistent with the standard spherical model. Decay rates for our two models, on 2D and 3D lattices with both ferromagnetic and antiferromagnetic exchange couplings, are the subjects of $\S \S 5,6$. Our findings are gathered and discussed in $\$ 7$. 


\section{Hamiltonians}

Classical spins, $\left\{\mathbf{S}_{a}\right\}$, are placed at sites, labelled by the index $a$, on a lattice with spatial dimension, $d$. An exchange interaction, of strength $J$, operates between nearest-neighbour spins. The $x-y$ model is defined by the Hamiltonian,

$$
\text { (I) } \quad \mathcal{H}=-J \sum_{a, \delta}\left(S_{a}^{x} S_{a+\delta}^{x}+S_{a}^{y} S_{a+\delta}^{y}\right)
$$

where $x$ and $y$ denote Cartesian components of the three dimensional spins, and the site labelled $(a+\delta)$ is one of $r$ bearest-neighbours to the site labelled $a$. Much the same notation is required for the fully isotropic Heisenberg model with the addition of a single-site magnetic anisotropy. We sometimes, for brevity, refer to this model as model-II, and it is defined by,

(II) $\quad \mathcal{H}=-J \sum_{a, \delta} \mathbf{S}_{a} \cdot \mathbf{S}_{a+\delta}-B \sum_{a}\left(S_{a}^{z}\right)^{2}$.

In (2.1) and (2.2), $J>0(<0)$ is referred to as a ferromagnetic (antiferromagnetic) exchange coupling. The anisotropy parameter in (2.2) takes all values; $B>0$ yields a uniaxial anisotropy, and $B<0$ yields a planar anisotropy that curbs out-of-plane (the $x-y$ plane) spin fluctuations.

\section{Coupled-Mode Equations}

The time-dependent spin relaxation function is denoted by $F(\mathbf{q}, t)$; it is formed from the autocorrelation of spatial Fourier components with wave vector $\mathbf{q}$ of the spin density. For quantities belonging to the in-plane correlations (defined to be the $x$ and $y$ Cartesian components of the spins) we attach a subscript zero, e.g. $F_{\mathrm{o}}(\mathbf{q}, t)$ is the spin relaxation function formed with the $x$-component of the spin density, which is identical with the relaxation function formed with the $y$-component of the spin density. Out-of-plane spin fluctuations ( $z-z$ spin autocorrelations) are described by $F(\mathbf{q}, t)$. 
The relaxation functions are calculated in terms of a memory function. For the out-ofplane fluctuations the memory function is denoted by $K(\mathbf{q}, t)$, and,

$$
\dot{F}(\mathbf{q}, t)=-\int_{0}^{t} \mathrm{~d} t^{\prime} F\left(\mathbf{q}, t^{\prime}\right) K\left(\mathbf{q}, t-t^{\prime}\right)
$$

Here, the dot on $F(\mathbf{q}, t)$ denotes a time derivative. A similar equation holds for $F_{\mathrm{o}}(\mathbf{q}, t)$ in which the memory function is $K_{0}(\mathbf{q}, t)$.

The coupled-mode approximations for $K(\mathbf{q}, t)$ and $K_{\mathrm{o}}(\mathbf{q}, t)$ are derived in the standard manner. In essence, $K(\mathbf{q}, t)$ is the relaxation function formed with the first time derivative of $S_{\mathbf{q}}^{z}$, which is derived by evaluating the commutator of $S_{\mathbf{q}}^{z}$ with the appropriate Hamiltonian. The relaxation function thereby obtained involves four spin operators. The final step, in deriving the coupled-mode approximation to $K(\mathbf{q}, t)$, is a particular closure of the equations of motion, achieved by a factorization of this relaxation function in the memory function in to products of two-spin relaxation functions, namely $F(\mathbf{q}, t)$ and $F_{\mathrm{o}}(\mathbf{q}, t)$. So, in the end, $F_{\mathrm{o}}(\mathbf{q}, t)$, for example, is expressed in terms of $K_{\mathrm{o}}(\mathbf{q}, t)$ which in the coupled-mode approximation is a spatial convolution of $F_{\mathrm{o}}(\mathbf{q}, t)$ and $F(\mathbf{q}, t)$; the memory function for $F(\mathbf{q}, t)$ contains $F_{\mathrm{o}}(\mathbf{q}, t)$ and not $F(\mathbf{q}, t)$. Thus, the equations for the spin relaxation function of immediate interest have the form of integral-differential equations, and $F(\mathbf{q}, t)$ and $F_{\mathrm{o}}(\mathbf{q}, t)$ are self-consistent solutions.

Following through the approximation scheme for the memory functions described in the foregoing paragraph we find the following key results.

\subsection{Model-I}

The memory functions for the $x-y$ model are,

$$
\chi(\mathbf{q}) K(\mathbf{q}, t)=T(2 r J / N)^{2} \sum_{\mathbf{p}}\left(\gamma_{\mathbf{q}-\mathbf{p}}-\gamma_{\mathbf{p}}\right)^{2} \chi_{\mathrm{o}}(\mathbf{p}) \chi_{\mathrm{o}}(\mathbf{q}-\mathbf{p}) F_{\mathrm{o}}(\mathbf{p}, t) F_{\mathrm{o}}(\mathbf{q}-\mathbf{p}, t)
$$

and, 


$$
\chi_{\mathrm{o}}(\mathbf{q}) K_{\mathrm{o}}(\mathbf{q}, t)=T(2 r J / N)^{2} \sum_{\mathbf{p}} \gamma_{\mathbf{p}}^{2} \chi_{\mathrm{o}}(\mathbf{p}) \chi(\mathbf{q}-\mathbf{p}) F_{\mathrm{o}}(\mathbf{p}, t) F(\mathbf{q}-\mathbf{p}, t)
$$

In these expressions, $\gamma_{\mathrm{q}}$ is a geometric factor that depends on the lattice dimensionality and its point group symmetry,

$$
r \gamma_{\mathbf{q}}=\sum_{\delta} \exp (\mathbf{i q} \cdot \delta)
$$

The other new quantities appearing in (3.2) and (3.3) are the wave vector-dependent isothermal susceptibilities for out-of-plane, $\chi(\mathbf{q})$, and in-plane, $\chi_{0}(\mathbf{q})$, spin fluctuations. These quantities are the subject of the following section.

\subsection{Model-II.}

The memory function for the out-of-plane fluctuations satisfies an equation that has exactly the same structure as (3.2), and the value for $\chi_{0}(\mathbf{q})$ appropriate for the Hamiltonian (2.2). Note that the memory function $K(\mathbf{q}, t)$ vanishes in the limit of long wavelengths. This property is a consequence of the fact that for both models the $z$-component of the spin density is a constant of motion.

The in-plane memory function for model-II is,

$$
\chi_{\mathrm{o}}(\mathbf{q}) K_{\mathrm{o}}(\mathbf{q}, t)=\left(T / N^{2}\right) \sum_{\mathbf{p}}\left\{2 r J\left(\gamma_{\mathbf{q}-\mathbf{p}}-\gamma_{\mathbf{p}}\right)+2 B\right\}^{2} \chi_{\mathrm{o}}(\mathbf{p}) \chi(\mathbf{q}-\mathbf{p}) F_{\mathrm{o}}(\mathbf{p}, t) F(\mathbf{q}-\mathbf{p}, t)
$$

In all these expressions, $T$ is the temperature measured in units of Boltzmann's constant, and $N$ is the number of spins on the lattice (equal to the number of lattice points). 


\section{Static Spin correlations}

The coupled-mode theory of spin fluctuations requires the isothermal susceptibilities as inputs. We have decided to use values obtained from the appropriate spherical models (Mattis 1985). Since the pairs of susceptibilities for our two anisotropic models, to the best of our knowledge, have not appeared in the literature it is prudent to discuss some of their features. We first outline our method for calculating the spin correlation functions and the susceptibilities, which follows an approach by Hubbard (1971) to the isotropic Heisenberg magnet.

In this approach, the defining equations are obtained from the $\mathrm{f}$-sum rule. This means that one uses short-time properties of the memory function, and the ensuing spin correlation functions are obtained by solving the defining equations for arbitrary wave vectors. In the context of the early development of the coupled-mode theory, such an approach might not seem entirely consistent. For, the coupled-mode theory of critical spin fluctuations was developed with the aim of describing the long-time behaviour of long wavelength fluctuations, i.e. the modes that witness the critical fluctuations. From this stance there is no good reason to be confident about the value of the theory at short times and short wavelengths, both of which enter the approach to calculating the static correlations from the f-sum rule. However, it has been shown that coupled-mode theory, of the variety used here, is very reliable at all wave vectors and temperatures, including an infinite temperature (Cuccoli et al. 1989, Lovesey et al. 1994). In our opinion this positive experience negates reservations about the use of the f-sum rule to obtain the static correlations. In the present case, where we need the in-plane and outof-plane susceptibilities the approach has the real advantage of providing mutually consistent expressions. Our view is that the models under discussion are defined to possess the static correlations of the appropriate spherical models.

Returning to the approach to static correlation functions based on the f-sum rule, the defining equations are derived from the identity,

$$
\ddot{F}(\mathbf{q}, 0)=-K(\mathbf{q}, 0)
$$


which follows from (3.1) and the normalization $F(\mathbf{q}, 0)=1$, and the f-sum rule,

$$
\chi(\mathbf{q}) \ddot{F}(\mathbf{q}, 0)=-\left\langle\left[\left[S^{z}(\mathbf{q}), \mathscr{H}\right], S^{z}(-\mathbf{q})\right]\right\rangle,
$$

where the angular brackets denote a thermal average. A similar set of relations exist for the inplane fluctuations, with the spatial Fourier component of the spin density $S^{z}(\mathbf{q})$ replaced by $S^{x}(\mathbf{q})$. The nested commutators in (4.2) are evaluated with the appropriate spin Hamiltonian $\left(\left[S^{\alpha}(\mathbf{q}), \mathscr{H}\right]\right.$ is required to get an expression for $\dot{S}^{\alpha}(\mathbf{q})$ which is the building block of the coupled-mode approximation to the memory function).

\subsection{Model-I}

On following through the programme outlined in the foregoing paragraphs, we find for the $x-y$ model,

$$
\chi(\mathbf{q})=N / \mu,
$$

and

$$
\chi_{\circ}(\mathbf{q})=N /\left(\mu-2 r J \gamma_{\mathbf{q}}\right)
$$

The parameter $\mu$ is related to the temperature. To this end, we demand,

$$
\left\langle\mathbf{S}_{a} \cdot \mathbf{S}_{a}\right\rangle=S(S+1)
$$

where $S$ is the magnitude of the spin variable. The spin correlations are provided by,

$$
\left\langle S_{a}^{z} S_{b}^{z}\right\rangle=\left(T / N^{2}\right) \sum_{\mathbf{q}} \chi(\mathbf{q}) \exp \left\{\mathbf{i q} \cdot\left(\mathbf{R}_{a}-\mathbf{R}_{b}\right)\right\}
$$


and a corresponding expression for $\left\langle S_{a}^{x} S_{b}^{x}\right\rangle=\left\langle S_{a}^{y} S_{b}^{y}\right\rangle$. On introducing the molecular field approximation to the critical temperature of the isotropic Heisenberg magnet, $T_{m}=2 r J S(S+1) / 3$, one finds,

$$
T\{(1 / \mu)+2 I(\mu / 2 r J)\}=3 T_{m}
$$

In this expression, $I(x)$ is the standard extended Watson integral,

$$
I(x)=(1 / N) \sum_{\mathbf{q}}\left(x-\gamma_{\mathbf{q}}\right)^{-1}
$$

For a square lattice,

$$
I(x)=(2 / \pi x) K(1 / x)
$$

where $K(y)$ is the complete elliptic integral of the first kind. It is not possible with $3 \mathrm{D}$ lattices to express $I(x)$ in terms of standard, tabulated functions; useful analytic results are given by Joyce (1972), and Mannari and Kawabata (1964) have prepared tables of numerical results for the three cubic lattices.

The result (4.3) might easily have been anticipated; it is the susceptibility for a perfect paramagnet and this is appropriate for the out-of-plane fluctuations since no exchange force exists between $z$-components of the spin in the $x-y$ model. The result (4.4) for the in-plane susceptibility, taken with (4.7) for the temperature scale, is the standard spherical model.

In the limit of high temperatures, $T \gg J, I(\mu) \sim(1 / \mu)$ and the temperature scale is no different from that for the isotropic Heisenberg model; $\mu \sim T$ for high temperatures. The smallest physically sensible value of the temperature parameter, $\mu$, is $2 r J$.

The integral $I(1)$ is finite for $3 \mathrm{D}$ lattice, and not finite for a $2 \mathrm{D}$ lattice. In the former case, the temperature scale (4.7) evaluated for $\mu=2 r J$ defines the critical temperature, $T_{\text {c. }}$ Table 1 
contains values of $T_{\mathrm{c}}$ for the three cubic lattices for $S=1 / 2$, together with corresponding values obtained from extensive series expansions. The level of agreement between the two sets of results is tolerable; one might reasonably expect the spherical model to be least reliable in the extreme quantum limit, $S=1 / 2$, and progressively more reliable with increasing values of $S$. Because $I(1)>1$, for the cubic lattices, we conclude that $T_{\mathrm{c}}(x-y)>T_{\mathrm{c}}$ (isotropic). For $S=1 / 2$ the same result is obtained from series expansions, cf. Table 1.

In the immediate vicinity of the critical temperature, the in-plane susceptibility takes on the form proposed by Ornstein and Zernike, namely,

$$
\chi_{\mathrm{o}}(q)=\frac{\left(N / 2 r J \rho^{2}\right)}{\left(\kappa_{\circ}^{2}+q^{2}\right)}
$$

Here, the inverse correlation length, $\kappa_{0}$, satisfies ( $\mu$ is measured in units of $2 r J$ ),

$$
\rho^{2} \kappa_{\circ}^{2}=(\mu-1) ; \operatorname{limit} \mu \rightarrow 1^{+},
$$

and the length $\rho$ is defined by the small -q expansion of the geometric factor,

$$
\gamma_{q}=1-\rho^{2} q^{2}+\cdots
$$

From (4.7) and the properties of the extended Watson integral in the limit $\mu \rightarrow 1^{+}$,

$$
\rho \kappa_{\mathrm{o}}=(\mu-1)^{1 / 2} \propto\left(T-T_{\mathrm{c}}\right) / T_{\mathrm{c}},
$$

and only the coefficient of proportionality differs for the $x-y$ and isotropic Heisenberg models. The coefficient is reduced in the $x-y$ model by a factor $(2 I(1)+1) / 3 I(1)$.

Turning to the equivalent analysis for a $2 \mathrm{D}$ lattice, the key relation is, 


$$
I(\mu) \rightarrow(2 / \pi) \ln \left\{\frac{2 \sqrt{2}}{(\mu-1)}\right\} ; \text { limit } \mu \rightarrow 1^{+} .
$$

We conclude that, there is no phase transition at a finite temperature, a finding that is in accord with the Mermin-Wagner (1966) theorem. If the inverse correlation length for the corresponding isotropic Heisenberg model is denoted by $\kappa_{\mathrm{i}}$, we find for $T \rightarrow 0$,

$$
\left(\kappa_{\mathrm{o}} / \kappa_{\mathrm{i}}\right)=\exp \left\{-\pi T_{m} / 4 T\right\}
$$

Thus, just as we found for $3 \mathrm{D}$, in $2 \mathrm{D}$ the reduction in spin isotropy increases the correlation length. The effect is still pronounced at moderate temperatures, e.g. with $\left\{T / 3 T_{\mathrm{m}}\right\}=0.259$, one finds $\rho \kappa_{0}=0.33$ and $\rho \kappa_{i}=0.42$.

\subsection{Model- II}

The two susceptibilities are,

$$
\chi_{\circ}(\mathbf{q})=N /\left(\mu_{\circ}-2 r J \gamma_{\mathbf{q}}\right)
$$

and,

$$
\chi(\mathbf{q})=N /\left(\mu-2 r J \gamma_{\mathbf{q}}\right)
$$

with

$$
\mu=\mu_{\mathrm{o}}-2 B \text {. }
$$

For the particular example of classical spins with one degree of freedom (classical Ising variables, or sticks, capable of assuming two discrete orientations) we have demonstrated that the conventional derivation of the spherical model, through use of the saddle point method to calculate the partition function, leads to (4.17) and the appropriate limit of the temperature scale 
(4.21). Looking at (4.16 - 4.18), for $B>0$ (axial anisotropy) the out-of-plane fluctuations become critical at $\mu=2 r J$, and at this temperature $\kappa_{\mathrm{o}}$ is finite, namely,

$$
\rho \kappa_{\circ}=(B / r J)^{1 / 2} \text {. }
$$

In the opposite case, $B<0$ (planar anisotropy) the properties of the in-plane and out-of-plane fluctuations are reversed.

The temperature scale is set by the relation $\left(\mu\right.$ and $\mu_{0}$ in units of $\left.2 r J\right)$,

$$
T\left\{2 I\left(\mu_{\mathrm{o}}\right)+I(\mu)\right\}=3 T_{\mathrm{m}}
$$

For $B>0$, the critical temperature is,

$$
T_{\mathrm{c}}\{2 I(1+2 b)+I(1)\}=3 T_{\mathrm{m}},
$$

where $b=B / 2 r J$. Since $I(x)$ is a monotonically decreasing function of its argument, we conclude that a finite uniaxial anisotropy increases the critical temperature above the value for the isotropic Heisenberg model. This is in line with physical intuition, since a uniaxial anisotropy effectively enhances the exchange interaction. For a given value of $|B|$, the critical temperature of the model with a planar anisotropy is intermediate between the values for the isotropic and uniaxial anisotropy models. This finding differs from that obtained in the molecular field approximation, where planar $(B<0)$ anisotropy reduces the critical temperature below $T_{m}$. In the extreme limit $|B| \rightarrow \infty$ our spherical model calculations give, $T_{\mathrm{c}}$ (isotropic) $<T_{\mathrm{c}}(x-y)<T_{\mathrm{c}}$ (planar) $<T_{\mathrm{c}}$ (axial) $<T_{m}$. Of course, in the molecular field approximation this particular limit has no real physical meaning since in this approximation $T_{\mathrm{c}}$ (planar) and $T_{\mathrm{c}}$ (axial) are unbounded, e.g. Lovesey (1987).

The in-plane and out-of-plane inverse correlation lengths are denoted by $\kappa_{0}$ and $\kappa$, respectively. The relation, 


$$
\rho^{2} \kappa^{2}=\rho^{2} \kappa_{o}^{2}-2 b,
$$

follows from (4.18).

For the $2 \mathrm{D}$ model, the ratio of the correlation lengths at low temperatures takes on extreme values. By low temperatures we mean $T \leq\left(T_{m} / 5\right)$. In this region, the "critical" mode has a very long correlation length whereas the "non-critical" correlation length saturates to a value determined by the anisotropy parameter, $B$. As an example, consider a uniaxial anisotropy, and take $b=(B / 2 r J)=0.09$ and $\left(T / T_{m}\right)=0.19$. We find, for this case $\left(\kappa / \kappa_{0}\right)=5.4 \cdot 10^{-9}$. 


\section{Decay Rates: Model-I}

We will analyse the dynamical correlations in our models in terms of decay rates derived for the coupled-mode equations provided in $\$ 3$. The decay rates for the in-plane and out-ofplane fluctuations are denoted by $\Gamma_{\mathrm{o}}(\mathbf{q})$ and $\Gamma(\mathbf{q})$, respectively.

From (3.2) and (3.3) we obtain,

$$
\chi(\mathbf{q}) \Gamma(\mathbf{q})=T(2 r J / N)^{2} \sum_{\mathbf{p}}\left(\gamma_{\mathbf{q}-\mathbf{p}}-\gamma_{\mathbf{p}}\right)^{2} \chi_{\circ}(\mathbf{p}) \chi_{\mathrm{o}}(\mathbf{q}-\mathbf{p}) /\left\{\Gamma_{\mathrm{o}}(\mathbf{p})+\Gamma_{\mathrm{o}}(\mathbf{q}-\mathbf{p})\right\}
$$

and,

$$
\chi_{\circ}(\mathbf{q}) \Gamma_{\mathrm{o}}(\mathbf{q})=T(2 r J / N)^{2} \sum_{\mathbf{p}} \gamma_{\mathbf{p}}^{2} \chi_{\circ}(\mathbf{p}) \chi(\mathbf{q}-\mathbf{p}) /\left\{\Gamma_{\mathrm{o}}(\mathbf{p})+\Gamma(\mathbf{q}-\mathbf{p})\right\}
$$

We are interested in long wavelength fluctuations, and therefore consider the limit $q \rightarrow 0$ in (5.1) and (5.2). Furthermore, we focus on the temperature interval immediately above and at the critical temperature (for $2 \mathrm{D}$ models the appropriate temperature range is $T \ll J$ ). The necessary condition is that the susceptibility for the critical mode is extremely large at the ordering wave vector.

Consider, for the moment, a 2D lattice and a ferromagnetic exchange coupling $(\mathrm{F})$; one finds from (5.1) and (5.2),

$$
\Gamma_{\mathrm{o}}(q)=A\left(\mathrm{\kappa}_{\circ}^{2}+q^{2}\right) f_{\mathrm{o}}(q),
$$

and

$$
\Gamma(q)=A q^{2} f(q)
$$

where for a spatial dimension $d$, 


$$
A^{2}=T\left(r J v_{\mathrm{o}}\right) / \pi^{d-1}
$$

and $v_{0}$ is the area (volume) of a primitive unit cell, e.g. square lattice $v_{0}=a_{0}^{2}$, and bodycentred-cubic (b.c.c) lattice $v_{\mathrm{o}}=a_{\mathrm{o}}^{3} / 2$ where $a_{\mathrm{o}}$ is the cell dimension. The functions $f_{\mathrm{o}}(q)$ and $f(q)$ satisfy the integral equations,

$$
f(q)=\int_{q}^{\infty} \frac{p^{3} \mathrm{~d} p}{\left(\kappa_{\mathrm{o}}^{2}+p^{2}\right)^{3} f_{\mathrm{o}}(p)}
$$

and

$$
f_{\mathrm{o}}(q)=\int_{q}^{\infty} \frac{p \mathrm{~d} p}{\left(\kappa_{\mathrm{o}}^{2}+q^{2}\right)\left\{p^{2} f(p)+\left(\kappa_{\mathrm{o}}^{2}+p^{2}\right) f_{\mathrm{o}}(p)\right\}}
$$

In arriving at (5.6) and (5.7) the upper limit of integration over the Brillouin has been extended to infinity. An inspection of the integral equations reveals that, in the limit $q \rightarrow 0 f_{0}(q)$ and $f(q)$ are both benign functions. Hence, a tolerable approximation is obtained by replacing the functions by their values at $q=0$. Our estimate for the decay rates of the $2 \mathrm{D}, \mathrm{F} x-y$ model are,

$$
\Gamma_{\mathrm{o}}(q)=0.621 A\left(\kappa_{\mathrm{o}}^{2}+q^{2}\right) / \kappa_{\mathrm{o}}
$$

and,

$$
\Gamma(q)=0.403 A q^{2} / \kappa_{\mathrm{o}}
$$

Recall that, for $d=2$ the quantity $A \propto T^{1 / 2}$. Even so, $\Gamma(q)$ increases with decreasing temperature since, in the limit of low temperatures,

$$
\rho \kappa_{\mathrm{o}}=(\mu-1)^{1 / 2} \sim 2 \sqrt{2} \exp \left\{-3 \pi T_{m} / 4 T\right\} .
$$


The corresponding value of $\Gamma_{0}(0)$ is very small.

The relations (5.3) and (5.4) are convenient also for the 3D lattice, where now one can reasonably set $T=T_{\mathrm{c}}$ in the quantity $A$, defined in (5.5). At the critical temperature, $\kappa_{\mathrm{o}}=0$, the integral equations for $f_{\mathrm{o}}(q)$ and $f(q)$, which correspond to (5.6) and (5.7) for $2 \mathrm{D}$, admit nontrivial solutions; $3 \mathrm{D}, \mathrm{F} \kappa_{0}=0$,

$$
\Gamma_{\mathrm{o}}(q)=0.816 A q^{3 / 2}
$$

and,

$$
\Gamma(q)=2 \Gamma_{\mathrm{o}}(q)
$$

On the other hand, for $\kappa_{0} \neq 0$ we obtain the estimate,

$$
\Gamma_{\circ}(q)=0.711 A\left(\kappa_{\circ}^{2}+q^{2}\right) / \kappa_{\mathrm{o}}{ }^{1 / 2},
$$

and

$$
\Gamma(q)=0.553 A q^{2} / \kappa_{\mathrm{o}}^{1 / 2}
$$

It is interesting to observe that, the critical properties of the $3 \mathrm{D}, \mathrm{F} x-y$ model are the same as those of the AF isotropic Heisenberg model.

For the $x-y$ model, the decay rates $\Gamma(q)$ and $\Gamma_{0}(q)$ are homogeneous functions of the form,

$$
q^{d / 2} R_{1}\left(q / \kappa_{\mathrm{o}}\right)
$$


where $R_{1}(x)$ is usually referred to as a scaling function. In this context, the foregoing results are the explicit forms of the scaling functions for in-plane and out-of-plane fluctuations in the extreme limits $x \rightarrow 0$ and $x \rightarrow \infty$. Applied to the 3D, $x-y$ model we conclude that the dynamic critical exponent $z=(d / 2)=3 / 2$. As we have remarked, this value for the exponent is the same as for the isotropic AF model (Hohenberg and Halperin 1977). The value $z=(d / 2)$ for the $x-y$ model is consistent with calculations based on the renormalization group method.

As the final topic in this section we consider the behaviour of the antiferromagnetically exchange coupled (AF) $x-y$ model. We remarked in the introduction that, the critical properties of the $x-y$ model are independent of the sign of the exchange coupling (Betts 1974). So, the following working, to some extent, is to confirm that our analysis is sound with respect to this aspect of the model.

Turning back to the in-plane susceptibility, equ. (4.4), we observe that after replacing $J$ by $-J$, the ordering wave vector is $\mathbf{w}$, which satisfies $\gamma_{\mathbf{q}+\mathbf{w}}=-\gamma_{\mathbf{q}}$. Thus, in equs. (5.1) and (5.2) for the decay rates it is convenient to expose the influence of the incipient AF ordering by shifting the summation variable $\mathbf{p} \rightarrow \mathbf{p}+\mathbf{w}$. Evidently, there is a coupling between fluctuations that take place near the Brillouin zone centre and at the ordering vector, $\mathbf{w}$. In consequence, we must construct a second pair of equations for the in-plane and out-of-plane fluctuations that occur in the vicinity of $\mathbf{w}$; we denote the corresponding decay rates by $\Gamma_{0}^{w}$ and $\Gamma^{w}$.

For a $2 \mathrm{D}$ lattice, we find,

$$
\Gamma_{\circ}=\Gamma^{w}=\left(A \zeta / \kappa_{\mathrm{o}}\right)
$$

where $\zeta$ is a function of $\kappa_{0}$, and determined by the equation,

$$
\zeta^{2}=\ln \left(1+\zeta / 0.621 \kappa_{\circ} \rho\right)
$$

In the limit $\mathrm{T} \rightarrow 0$, 


$$
\Gamma_{\mathrm{o}}=\Gamma^{w}=4 \sqrt{2} J
$$

The value of $\Gamma(q)$ is identical to the same function in the ferromagnetic model, and $\Gamma_{\mathrm{o}}^{w}(q)=\Gamma_{\mathrm{o}}(q)$. These are the results we anticipated.

Finally, we mention our results for the decay rates of the 3D, AF $x-y$ model at $T=T_{\mathrm{c}}$. These are,

$$
\Gamma_{\mathrm{o}}=\Gamma^{w}=3.610 \mathrm{~A} / \rho^{3 / 2},
$$

together with $\Gamma(q)=2 \Gamma_{\mathrm{o}}^{w}(q)$ and $\Gamma_{\mathrm{o}}^{w}(q)$ is identical to $\Gamma_{\mathrm{o}}(q)$ for the $3 \mathrm{D}, \mathrm{F} x$-y model given in (5.10).

\section{Decay Rates: Model-II}

The method of analysis we use for the Heisenberg model supplemented by a single-site anisotropy, denoted by model-II, is the same as that applied in the previous section to the $x-y$ model. In view of this, in this part of our report we do not give details of our working, and focus on the results. It is convenient to employ the non-universal, material constant $A$ defined in equ (5.5).

A way of expressing the basic differences between two models is to contrast the homogeneous functions for the decay rates. For model-I, we established that the decay rates can be expressed in the form $q^{d / 2} R_{1}\left(q / \kappa_{0}\right)$, where $R_{1}(x)$ is a so-called scaling function. Extending this concept to the decay rates for a ferromagnetically coupled model-II, as proposed by Riedel and Wegner (1970), we have established that the in-plane and out-of-plane decay rates are of the form,

$$
q^{(d+2) / 2} R_{2}\left(q / \kappa, q / \kappa_{\mathrm{o}}\right) .
$$


For a 3D lattice, the dynamic critical exponent $z=(d+2) / 2=5 / 2$ is the same as for the isotropic F model. We turn now to the task of obtaining the interesting limiting values of the scaling function for 2D and 3D lattices.

For a 2D lattice and a ferromagnetic exchange coupling, the in-plane and out-of-plane decay rates are,

$$
\Gamma_{\mathrm{o}}(q)=\frac{1}{2} A \rho\left(\kappa_{\circ}^{2}+q^{2}\right) \xi^{1 / 2}
$$

and

$$
\Gamma(q)=\frac{1}{2} A \rho q^{2}\left(\kappa^{2}+q^{2}\right) / \kappa_{\mathrm{o}}^{2} \xi^{1 / 2}
$$

where $\kappa$ and $\kappa_{0}$ are the out-of-plane and in-plane inverse correlation lengths, respectively, and we define $\theta=\kappa / \kappa_{0}$. The function $\xi$ in (6.1) satisfies,

$$
\xi=-(1+\xi) \ln \theta^{2}+(\xi-1) \ln \left(\theta^{2} / \xi\right)+\left[(1+\xi)\left(\theta^{2}-1\right)+(1-\xi)^{2}\right] Q(\theta, \xi),
$$

where for $a=\left\{\left(\xi+\theta^{2}\right)^{2}-4 \xi\right\}>0$ the function $Q(\theta, \xi)$ is,

$$
Q(\theta, \xi)=a^{-1 / 2} \ln \left\{\frac{1}{4 \xi}\left[\left(\xi+\theta^{2}\right)+a^{1 / 2}\right]^{2}\right\}
$$

and for $b=\left\{4 \xi-\left(\xi+\theta^{2}\right)^{2}\right\}>0$,

$$
Q(\theta, \xi)=\left(2 / b^{1 / 2}\right) \tan ^{-1}\left\{b^{1 / 2} /\left(\xi+\theta^{2}\right)\right\}
$$

For the planar anisotropy, $\Gamma_{\mathrm{o}}(0)$ is very small and $\left\{\Gamma(q) / q^{2}\right\}$ very large in the limit of low temperatures, $\theta=\left(\kappa / \kappa_{0}\right) \rightarrow \infty$. In this limit, $\xi^{1 / 2} \rightarrow 1.241 \theta$, and,

$$
\Gamma_{\mathrm{o}}(0)=0.621 A \rho \kappa \kappa_{\mathrm{o}}
$$


and,

$$
\left\{\Gamma(q) / q^{2}\right\}=0.402 A \rho\left(\kappa / \kappa_{\mathrm{o}}\right)
$$

where $\rho \kappa=\sqrt{ }(2 b)$.

Looking next at the case of axial anisotropy, the low temperature limit corresponds to $\theta \rightarrow 0$, and here $\xi$ satisfies,

$$
\xi=\ln \left(\xi / \theta^{4}\right) .
$$

$\Gamma_{0}(0)$ decreases and $\left\{\Gamma(q) / q^{2}\right\}$ increases with increasing temperature. Representative examples of the temperature dependence of the decay rates are illustrated in fig. (1). In viewing the results bear in mind that the calculations do not involve explicitly the temperature scale set by the spherical model; the latter enters only in so far as we use the susceptibilities (4.16) and (4.17) in the construction of the integral equations for $\Gamma_{\mathrm{o}}(q)$ and $\Gamma(q)$.

Next on the agenda is the $3 \mathrm{D}, \mathrm{F}$ version of model-II. For the planar model at $T_{\mathrm{c}}, \rho \kappa=\sqrt{ }(2 \mathrm{~b})$ and,

$$
\begin{aligned}
& \Gamma_{\mathrm{o}}(q)=0.816 A \rho q^{3 / 2} \kappa, \\
& \Gamma(q)=1.632 A \rho q^{3 / 2}\left(\kappa^{2}+q^{2}\right) / \kappa .
\end{aligned}
$$

It is interesting to observe that these decay rates depend explicitly on the anisotropy parameter. For $\kappa_{0}>0$, the decay rates are,

$$
\begin{aligned}
& \Gamma_{\mathrm{o}}(q)=0.626 A \rho\left(\kappa_{\mathrm{o}}^{2}+q^{2}\right) \kappa_{\mathrm{o}}^{1 / 2} \xi^{1 / 2}, \\
& \Gamma(q)=0.626 A \rho q^{2}\left(\kappa^{2}+q^{2}\right) / \kappa_{\mathrm{o}}^{3 / 2} \xi^{1 / 2},
\end{aligned}
$$


where the dimensionless function $\xi$ satisfies,

$$
\theta=\frac{3}{4} \xi+\left[\theta^{2}+\xi^{1 / 2}\left(1-\xi^{1 / 2}-\xi\right)\right]\left\{\theta^{2}+2 \xi^{1 / 2}+\xi\right\}^{-1 / 2}
$$

In the limit $\theta=\left(\kappa / \kappa_{0}\right) \rightarrow \infty, \xi^{1 / 2} \rightarrow 1.134 \theta$ and,

$$
\begin{aligned}
& \Gamma_{\mathrm{o}}(0)=1.004 A b^{1 / 2} \kappa_{\mathrm{o}}{ }^{3 / 2} \\
& \left\{\Gamma(q) / q^{2}\right\}=0.781 A b^{1 / 2} / \kappa_{\mathrm{o}}{ }^{1 / 2} .
\end{aligned}
$$

These results and the results (6.5) are two limiting cases of the scaling behaviour contained in the general $\left(\kappa_{0}>0\right)$ relation $(6.6)$.

The corresponding results for axial anisotropy follow from (6.6) by noting that for $\theta=0$ the solution of $(6.7)$ is $\xi=1.770$. Hence, we conclude that for axial anisotropy the critical dynamics is conventional.

To illustrate the temperature dependence of $\Gamma_{0}(0)$, and $\left\{\Gamma(q) / q^{2}\right\}$ evaluated for $q \rightarrow 0$, it is useful to write the results in the form,

$$
\begin{aligned}
& \Gamma_{\circ}(0)=1.489 A \rho b^{5 / 4} \xi^{1 / 2} /\left|\theta^{2}-1\right|^{5 / 4}, \\
& \left\{\Gamma(q) / q^{2}\right\}=0.744 A \rho^{1 / 2} b^{1 / 4} \theta^{2} /\left(\xi^{1 / 2}\left|\theta^{2}-1\right|^{1 / 4}\right),
\end{aligned}
$$

where, as before, $b=|B / 2 r J|$, and the length $\rho$ is defined through (4.12). So, the temperature dependence of $\Gamma_{\mathrm{o}}(0)$ and $\left\{\Gamma(q) / q^{2}\right\}$ is given by,

$$
\Gamma_{\mathrm{o}}(0) \propto \xi^{1 / 2} /\left|\theta^{2}-1\right|^{5 / 4} \propto\left(\rho \kappa_{\mathrm{o}}\right)^{5 / 2} \xi^{1 / 2}
$$


and,

$$
\left\{\Gamma(q) / q^{2}\right\} \propto \theta^{2} /\left(\xi^{1 / 2}\left|\theta^{2}-1\right|^{1 / 4}\right) \propto(\rho \kappa)^{2} /\left\{\left(\rho \kappa_{\circ}\right)^{3 / 2} \xi^{1 / 2}\right\} .
$$

These functions are plotted in figs. (2) and (3). The non-universal material constant, $A$, is assumed to be a constant, and its value is given by (5.5) evaluated at $T=T_{\mathrm{c}}$. For axial anisotropy the in-plane decay rate $\Gamma_{0}(0)$ increases and the out-of-plane $\left\{\Gamma(q) / q^{2}\right\}$ decreases as $\theta=\left(\kappa / \kappa_{0}\right)$ decreases, i.e. as the temperature approaches $T_{\mathrm{c}}$. For planar anisotropy, the temperature dependence is slightly more complicated, but the limiting behaviour, $\theta^{-1} \rightarrow 0$, is the reverse of that found for axial anisotropy, with $\left\{\Gamma(q) / q^{2}\right\}$ increasing and $\Gamma_{\mathrm{o}}(0)$ decreasing as $T \rightarrow T_{\mathrm{c}}$ for $B<0$.

Finally, we turn to the behaviour of the decay rates for an antiferromagnetic exchange coupling. As in the case of the $x-y$ model, the number of decay rates is doubled on changing from $\mathrm{F}$ to $\mathrm{AF}$, since it is necessary to consider modes around the centre of the Brillouin zone, as with $\mathrm{F}$, and the wave vector, $\mathbf{w}$, that defines the incipient $\mathrm{AF}$ order. The main interest is the case of AF axial anisotropy, for the critical dynamics with planar anisotropy is the same for $\mathrm{F}$ and $\mathrm{AF}$.

For a 2D lattice and an antiferromagnetic exchange coupling the decay rates are conveniently expressed as,

$$
\begin{aligned}
& \Gamma(q)=2 A q^{2} g / \kappa_{\mathrm{o}}, \\
& \Gamma^{w}(q)=2 A\left(\kappa^{2}+q^{2}\right) g^{w} / \kappa_{\mathrm{o}}, \\
& \Gamma_{\mathrm{o}}=2 A \kappa_{\mathrm{o}} g_{\mathrm{o}},
\end{aligned}
$$

and, 


$$
\Gamma_{\mathrm{o}}^{w}(q)=2 A\left(\kappa_{\mathrm{o}}^{2}+q^{2}\right) g_{\mathrm{o}}^{w} / \kappa_{\circ}
$$

Here, as in $\S 5, \Gamma^{w}\left(\Gamma_{o}^{w}\right)$ is the decay rate for the out-of-plane (in-plane) fluctuations for a wave vector $\mathbf{q}$ measured relative to the incipient antiferromagnetic ordering wave vector, $\mathbf{w}$. The dimensionless amplitudes in (6.11) satisfy,

$$
\begin{aligned}
& g g_{\circ}^{w}=(1+\mu) / 16, \\
& g_{\circ} g^{w}=\ln \left(1+g_{\circ} / g_{\circ}^{w}\right) /\left\{2\left(1+\mu_{\circ}\right)\right\}, \\
& \left(g_{\circ}^{w}\right)^{2}=(1+\mu) /\left\{16\left(\exp \left[\frac{(1+\mu)^{2}}{8(1-b)^{2}}\right]-1\right)\right\},
\end{aligned}
$$

and,

$$
g_{\circ} g_{\circ}^{w}=\frac{1}{8}\left(1+\mu_{\circ}\right)\left\{-\ln \theta^{2}+\left(1+g_{\circ}^{w} / g^{w}\right) \ln \left(\frac{\theta^{2}+g_{\circ}^{w} / g^{w}}{1+g_{\circ}^{w} / g^{w}}\right)\right\} .
$$

The sign of $b=B / 2 r J$ in these equations corresponds to a uniaxial anisotropy. Amplitudes for the planar anisotropy follow by changing the sign of $b$ in (6.12) and in the relation between $\mu$ and $\mu_{0},(4.18)$.

For the latter case it can be shown that, in the limit $\kappa_{0} \rightarrow 0$,

$$
\left\{\Gamma(q) / q^{2}\right\} \rightarrow 0.570 A(1+b)^{1 / 2} / \kappa_{0}
$$

and

$$
\Gamma_{0}^{w}(0) \rightarrow 0.878 A(1+b)^{1 / 2} \kappa_{0} .
$$


The full temperature dependence of these decay rates, and $\Gamma_{0}$ and $\Gamma^{w}(q)$ are illustrated in fig. 4. Corresponding results for a uniaxial anisotropy are given in fig. 5 .

Turning to the $3 \mathrm{D}, \mathrm{AF}$ model - II we find at the critical temperature,

$$
\Gamma(q)=2 \Gamma_{0}^{w}(q)
$$

with,

$$
\Gamma_{0}^{w}(q)=1.155 A(1+b)^{1 / 2} q^{3 / 2}
$$

These results are for a planar anisotropy, and $b$ is the magnitude of $(B / 2 r J)$. With a uniaxial anisotropy the critical dynamics is conventional and the next set of equations apply.

Following the presentation of the $2 \mathrm{D}$ decay rates we choose to write,

$$
\begin{aligned}
& \Gamma(q)=2 A q^{2} g / \kappa_{\mathrm{o}}^{1 / 2}, \\
& \Gamma^{w}(q)=2 A\left(\kappa^{2}+q^{2}\right) g^{w} / \kappa_{\mathrm{o}}{ }^{1 / 2}, \\
& \Gamma_{\mathrm{o}}=2 A g_{\mathrm{o}} \kappa_{\mathrm{o}}{ }^{3 / 2},
\end{aligned}
$$

and,

$$
\Gamma_{\mathrm{o}}^{w}(q)=2 A\left(\kappa_{\mathrm{o}}^{2}+q^{2}\right) g_{\mathrm{o}}^{w} / \kappa_{\mathrm{o}}^{1 / 2}
$$

in which the dimensionless amplitudes are derived from,

$$
g g_{\circ}^{w}=\pi(1+\mu) / 32,
$$




$$
\begin{aligned}
& g^{w} g_{\circ}^{w}=\left\{\pi / 2\left(1+\mu_{\mathrm{o}}\right)\right\}\left[1+\left(1+g_{\circ} / g_{\circ}^{w}\right)^{1 / 2}\right]^{-1}, \\
& g_{\circ}^{w}\left(g_{\circ}^{w}+g\right)=\left\{\pi(1-b)^{2} / 2(1+\mu)\right\}\left[1+\left(\frac{g_{\circ}^{w}}{g_{\circ}^{w}+g}\right)^{1 / 2}\right]^{-1},
\end{aligned}
$$

and

$$
g_{\circ}\left(g_{\circ}^{w}+g^{w}\right)=\left\{\pi\left(1+\mu_{\circ}\right) / 8\right\}\left[\frac{(1-\theta)^{2}(1+\theta)}{(1+a)(\theta+a)}\right]
$$

where $\theta=\left(\kappa / \kappa_{0}\right)$ and,

$$
a^{2}=\left(1+\theta^{2} g^{w} / g_{\circ}^{w}\right) /\left(1+g^{w} / g_{0}^{w}\right)
$$

In these equations the sign of $b$ corresponds to a uniaxial anisotropy.

Figs 6 and 7 contain representative values of the decay rates (6.15) plotted against the appropriate critical inverse correlation length. The trends displayed in these two figures are readily understood from the defining equations and (6.16). For the uniaxial anisotropy the amplitudes are benign functions of $\kappa$, as anticipated. In the case of a planar anisotropy all amplitudes bar $g_{\mathrm{o}}$ are benign; for $\kappa_{\mathrm{o}} \rightarrow 0$ the amplitude $g_{\mathrm{o}}$ grows strongly so that $\Gamma_{\mathrm{o}}$ increases. 


\section{Conclusions and Discussion}

By way of orientation to the special features of the two anisotropic models we have discussed in the text we record the decay rates for the isotropic Heisenberg magnet. The decay rate for this model is denoted by $\Gamma_{i}(q)$. For a ferromagnetically $(\mathrm{F})$ coupled 3D magnet at $T_{c}$,

$$
\Gamma_{i}(q)=\frac{2}{3} A \rho q^{5 / 2},
$$

For $\mathrm{q} \ll<\kappa$,

$$
\Gamma_{i}(q)=\frac{1}{2}(\pi / 6)^{1 / 2} A \rho q^{2} \kappa^{1 / 2},
$$

and the corresponding result for the $2 \mathrm{D}$ lattice is,

$$
\Gamma_{i}(q)=\frac{1}{2} A p q^{2}
$$

Turning to the isotropic antiferromagnetically (AF) coupled isotropic Heisenberg magnet one finds for a three dimensional lattice and a temperature $=T_{c}$,

$$
\Gamma_{i}(q)=2.309 A q^{3 / 2},
$$

and

$$
\Gamma_{i}^{w}(q)=\frac{1}{2} \Gamma_{i}(q)
$$

where $\Gamma_{i}^{w}(q)$ is the decay rate for fluctuations near the antiferromagnetic ordering wave vector, w. The corresponding results for $T>T_{c}$ and $q \ll \kappa$ are,

$$
\Gamma_{i}(q)=0.782 A q^{2} / \kappa^{1 / 2},
$$


and,

$$
\Gamma_{i}^{w}(q)=1.005 A\left(\kappa^{2}+q^{2}\right) / \kappa^{1 / 2} .
$$

Lastly, for a two dimensional AF, in the limit of very low temperatures,

$$
\Gamma_{i}(q)=0.570 A q^{2} / \kappa,
$$

and,

$$
\Gamma_{i}^{w}(q)=0.878 A\left(\kappa^{2}+q^{2}\right) / \kappa .
$$

Here, and in all other results in this and the preceding sections, the material constant, $A$, is defined in (5.5).

The main outcome of the reported work on anisotropic models are explicit results for the decay rates. In the case of 3D models we have, in the general case, examined separately the critical domain $\left(T=T_{\mathrm{c}}\right.$ ) and the non-critical domain. On the other hand, for 2D systems no such distinction arises because there is no long-range order at a finite temperature.

In all, there is quite a large number of special cases of interest. Those not explicitly covered in our discussions can be deduced from the provided formulae. In a good few cases, simple algebraic results are not obtainable and the formulae must be solved numerically. This is particularly the case for model-II with an antiferromagnetic exchange coupling. We have just reported a few representative examples. To facilitate finding results, and making comparisons between properties of the various models, we have gathered some results in Tables 2 - 4. Here, and in most other places, we have expressed results in terms of the inverse correlation lengths. Thus, the results do not depend explicitly on spherical model temperature scales.

Use of decay rates, or the so-called Markovian approximation, is something of a coarse measure of the properties of long wavelength spin fluctuations. It tells us nothing which is reliable about the shape of the power spectrum. So, while they are valuable guides to the basic statistical mechanics of a model, a confrontation between experimental data and theoretical 
findings will most likely require more accurate information on the spectrum if it is to be really meaningful. In the context of the present study this means finding full solutions of the coupledmode equations provided in $\$ 3$ by numerical analysis.

\section{Acknowledgements}

We thank Dr. Arianna Montorsi for a valuable discussion on the static properties of the 2D models we have examined. Part of the work was accomplished while S.W.L. was a guest at the Aspen Center for Physics, Colorado. E.B. is grateful to the Associate Director and his staff at the DRAL, Rutherford Appleton Laboratory, for support during the final phase of the work.

Table 1

$\begin{array}{llll}\left(T_{\mathrm{c}} / J\right) \quad \text { s.c. } & \text { b.c.c } & \text { f.c.c }\end{array}$

\begin{tabular}{|c|c|c|c|c|}
\hline Spherical & $x-y$ model & 2.232 & 3.169 & 4.879 \\
\hline model & isotropic & 1.978 & 2.871 & 4.4 \\
\hline Series & $x-y$ model & 2.020 & 2.908 & \\
\hline expansion & isotropic & 1.68 & 2.53 & \\
\hline
\end{tabular}

Values of the critical temperature of the 3D $x-y$ spherical model for $S=1 / 2$, calculated from (4.7). The corresponding results obtained using high temperature series expansions are taken from the review article by Betts (1974). Results are given for the three cubic lattices. Results for the isotropic Heisenberg magnet $(S=1 / 2)$ are included for comparison. NB, the critical exponent $v$, for the inverse correlation length, has the value $1(2 / 3)$ for the spherical model $(S=1 / 2 x-y$ model $)$, and $\eta=0$. 
Table 2

\section{Isotropic Model}

$2 \mathrm{D}, q<<\kappa$

(F)

$\Gamma_{i} \sim \rho q^{2}$

$3 \mathrm{D}, T=T_{\mathrm{c}}$
(AF)

$\Gamma_{i} \sim q^{2} / \kappa$

$\Gamma_{i}^{w} \sim \kappa$

$$
\begin{aligned}
& \Gamma_{i} \sim q^{3 / 2} \\
& \Gamma_{i}^{w}=\frac{1}{2} \Gamma_{i}
\end{aligned}
$$

Decay rates for two (2D) and three (3D) dimensional lattices, and ferro (F) and antiferromagnetic coupling. The Hamiltonian for the spin system is given by (2.2) with $B=0$. 
Table 3

$x-y$, Model $-\mathbf{I}$

$2 \mathrm{D}, q<\mathrm{\kappa}_{0}$

(F)

(AF)

$$
\begin{aligned}
& \Gamma \sim q^{2} / \kappa_{\mathrm{o}} \\
& \Gamma_{\mathrm{o}} \sim \kappa_{\mathrm{o}}
\end{aligned}
$$

$$
\begin{aligned}
& \Gamma=\Gamma(F) \\
& \Gamma_{\mathrm{o}}^{w}=\Gamma_{\mathrm{o}}(\mathrm{F})
\end{aligned}
$$

$3 \mathrm{D}, T=T_{\mathrm{c}}$

$$
\begin{aligned}
& \Gamma=2 \Gamma_{0} \\
& \Gamma_{0} \sim q^{3 / 2}
\end{aligned}
$$

$$
\begin{aligned}
& \Gamma=2 \Gamma_{\circ}^{w}=\Gamma(\mathrm{F}) \\
& \Gamma_{\mathrm{o}}^{w}=\Gamma_{\mathrm{o}}(\mathrm{F})
\end{aligned}
$$

Decay rates for the $x-y$ model. The notation in the right-hand column for decay rates for ferromagnetic coupling is $\Gamma(F)$ or $\Gamma_{0}(F)$, e.g. the out-of-plane (AF) and (F) decay rates are the same, in the appropriate limits. 


\section{Table 4}

\section{planar, Model - II}

$2 \mathrm{D}, q<<\kappa_{0}$

(F)

$$
\begin{aligned}
& \left\{\Gamma(q) / q^{2}\right\} \sim \rho \kappa / \kappa_{0} \\
& \Gamma_{0}(0) \sim \rho \kappa \kappa_{0}
\end{aligned}
$$

\section{(AF)}

$\left\{\Gamma(q) / q^{2}\right\} \sim 1 / \kappa_{\mathbf{o}}$

$\Gamma_{\mathrm{o}}^{w}(0) \sim \kappa_{\mathrm{o}}$

$3 \mathrm{D}, T=T_{\mathrm{c}}$$$
\Gamma=2 \Gamma_{\mathrm{o}}
$$$$
\Gamma_{\mathrm{o}} \sim \rho q^{3 / 2} \kappa
$$
$\Gamma=2 \Gamma_{0}$
$\Gamma=2 \Gamma_{\mathrm{o}}^{w}$
$\Gamma_{\mathrm{o}}^{w}=q^{3 / 2}(1+b)^{1 / 2}$

Decay rates for the planar version of model-II with ferro-, and antiferromagnetic exchange coupling. Additional results are provided in $\$ 6$. 


\section{Figure Captions}

1. The temperature dependence of $\Gamma_{0}(0)$ and $\left\{\Gamma(q) / q^{2}\right\}$ for $2 \mathrm{D}, \mathrm{F}$ uniaxial model-II with $\mathrm{B}>0$. The quantities plotted as a function of the inverse correlation length, $\kappa$, are $\Gamma_{0} \propto\left(\rho \kappa_{0}\right)^{2} \xi^{1 / 2}$ and $\left\{\Gamma(q) / q^{2}\right\} \propto\left(\theta^{2} / \xi^{1 / 2}\right)$ where $\theta=\left(\kappa / \kappa_{0}\right)$ and $\xi$ is determined by $(6.2)$.

2. The variation of $\Gamma_{0}(0)$ and $\left\{\Gamma(q) / q^{2}\right\}$ for the $3 \mathrm{D}, \mathrm{F}$ model-II with $\kappa$, and two values of $\mathrm{B}>0$. The displayed quantities are $\Gamma_{\mathrm{o}}(0) \propto\left(\rho \kappa_{0}\right)^{5 / 2} \xi^{1 / 2}$ and $\left\{\Gamma(q) / q^{2}\right\} \propto\left\{(\rho \kappa)^{2} / \xi^{1 / 2}\left(\rho \kappa_{0}\right)^{3 / 2}\right\}$ and $\xi$ is determined by (6.7).

3. A comparative study of the temperature dependences of the decay rates for planar and uniaxial anisotropy in the 3D, F model-II. The functions displayed are $\Gamma_{\mathrm{o}}(0) \propto \xi^{1 / 2} /\left|\theta^{2}-1\right|^{5 / 4}$ and $\left\{\Gamma(q) / q^{2}\right\} \propto \theta^{2} /\left(\xi^{1 / 2}\left|\theta^{2}-1\right|^{1 / 4}\right)$, plotted as functions of $\theta^{-1}$ (planar) and $\theta$ (axial, c.f. fig. 2).

4. Illustrated is the temperature dependence of the decay rates for the $2 \mathrm{D}, \mathrm{AF}$ planar model-II with $|b|=0.10$. The quantities plotted against $\rho \kappa_{\mathrm{o}}$ are: (a) $\left\{\Gamma(q) / q^{2}\right\} \sim\left(g / \rho \kappa_{0}\right)$, (b) $\Gamma^{w}(0) \sim\left\{(\rho \kappa)^{2} g^{w} / \rho \kappa_{0}\right\}$, (c) $\Gamma_{\mathrm{o}} \sim\left(\rho \kappa_{0} g_{\mathrm{o}}\right)$ and (d) $\Gamma_{\mathrm{o}}^{w}(0) \sim\left(\rho \kappa_{\mathrm{o}} g_{\mathrm{o}}^{w}\right)$.

5. Results which correspond to fig. 4 but here for a uniaxial anisotropy $b=0.20$, and functions plotted against $\rho \kappa$.

6. Illustrated is the temperature dependence of the decay rates for the $3 \mathrm{D}$, AF planar model-II with $|b|=0.20$. The quantities plotted against $\rho \kappa_{\mathrm{o}}$ are: (a) $\left\{\Gamma(q) / q^{2}\right\} \sim g /\left(\rho \kappa_{\mathrm{o}}\right)^{1 / 2}$, (b) $\Gamma^{w}(0) \sim\left\{(\rho \kappa)^{2} g^{w} /\left(\rho \kappa_{0}\right)^{1 / 2}\right\},(c) \Gamma_{o} \sim\left(\rho \kappa_{0}\right)^{3 / 2} g_{0}$, and $(d) \Gamma_{o}^{w}(0) \sim\left(\rho \kappa_{o}\right)^{3 / 2} g_{o}^{w}$.

7. Results which correspond to fig. 6 but here for a uniaxial anisotropy $b=0.20$, and functions plotted against the critical inverse correlation length, $\kappa$. 


\section{References}

Bagnuls, C., and Joukoff-Piette, C., Phys. Rev. B11 (1975) 1986.

Berenzisnkii, V.L., Soviet Phys. JETP 32 (1971) 493.

Betts, D. D., in Phase Transitions and Critical Phenomena, vol. 3, edited by C. Domb and M. S. Green (Academic Press, London, 1974).

Collins, M. F., Magnetic Critical Scattering (O.U.P. New York, 1989).

Cowley, R. A., in Methods of Experimental Physics, vol. 23 part C (Academic Press, Orlando, 1987).

Cuccoli, A., Tognetti, V., and Lovesey, S.W., Phys. Rev. B39 (1989) 2619.

Hohenberg, P.C., and Halperin, B. I., Rev. Mod. Phys. 49 (1977) 435.

Hubbard, J., J. Phys. C4 (1971) 53.

Joyce, G. S., in Phase Transitions and Critical Phenomena, Vol. 2, edited by C. Domb and M. S. Green (Academic Press, London, 1972).

Kawasaki, K., in Phase Transitions and Critical Phenomena, Vol. 5A, edited by C. Domb and M. S. Green (Academic Press, London, 1976).

Kosterlitz, J. M., and Thouless, D. J., J. Phys. C6 (1973) 1181.

Landau, D.P., J. Appl. Phys. 73 (1993) 6091.

Lovesey, S. W., Condensed Matter Physics: Dynamic Correlations, 2nd edition, Vol. 61 in Frontiers in Physics (Benjamin/Cummings, Menlo Park, 1986).

Lovesey, S. W., Theory of Neutron Scattering by Condensed Matter, Vol. 2, 3rd edition (O.U.P., Oxford, 1987).

Lovesey, S. W., Engdahl, E., Cuccoli, A., Tognetti, V., and Balcar, E., J. Phys.: Condens. Matter, 6 (1994) L521.

Mattis, D. C., The Theory of Magnetism II (Springer-Verlag, Berlin, 1985).

Mannari, I., and Kawabata, C., Extended Watson Integrals and their Derivatives (Research Notes of the Dept. of Physics, Okayama University, 1964).

Mermin, N. D., and Wagner, H., Phys. Rev. Lett. 17 (1966) 1133.

Riedel, E., and Wegner, F., Phys. Rev. Lett. 24 (1970) 730. 


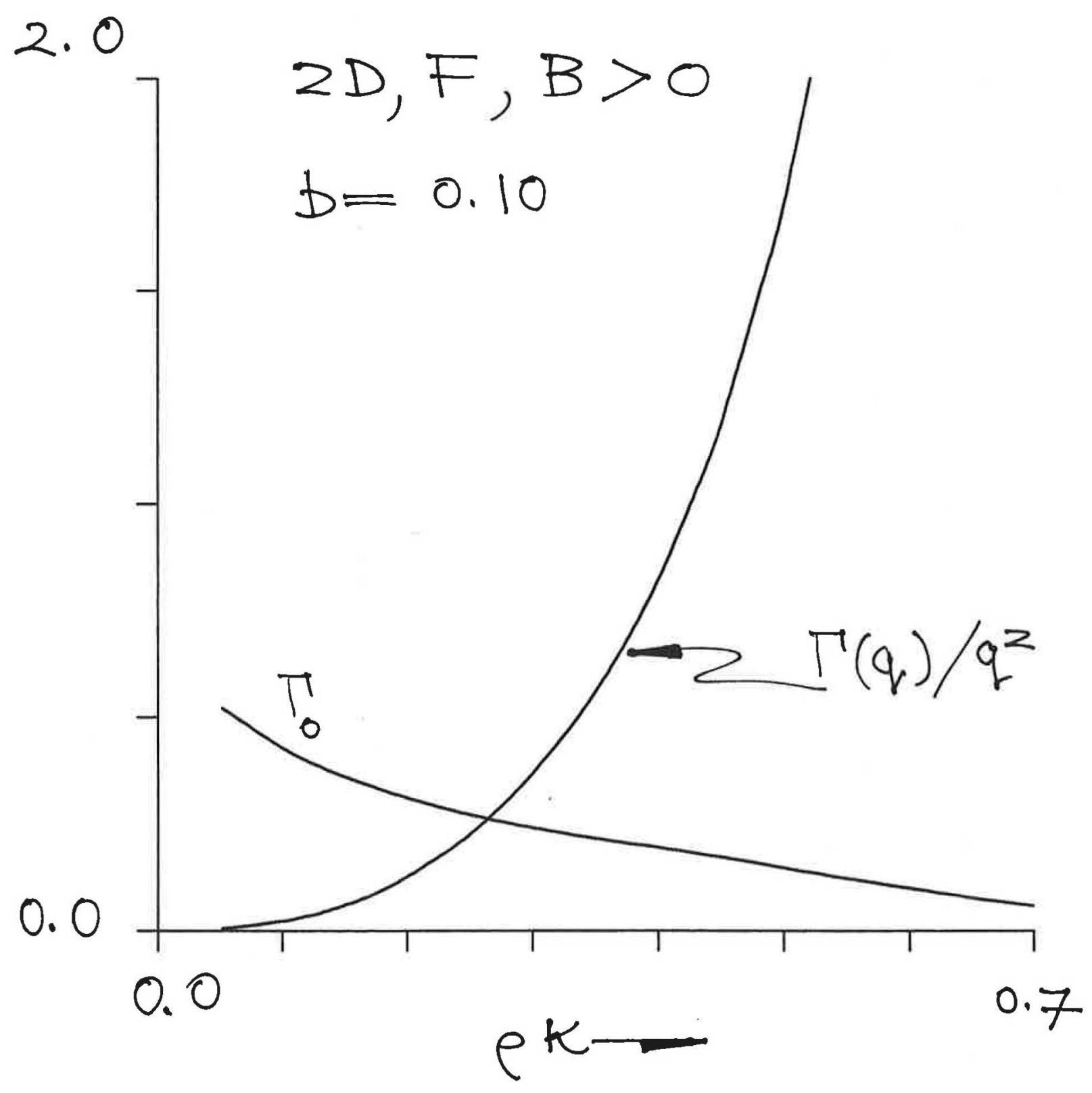

la 


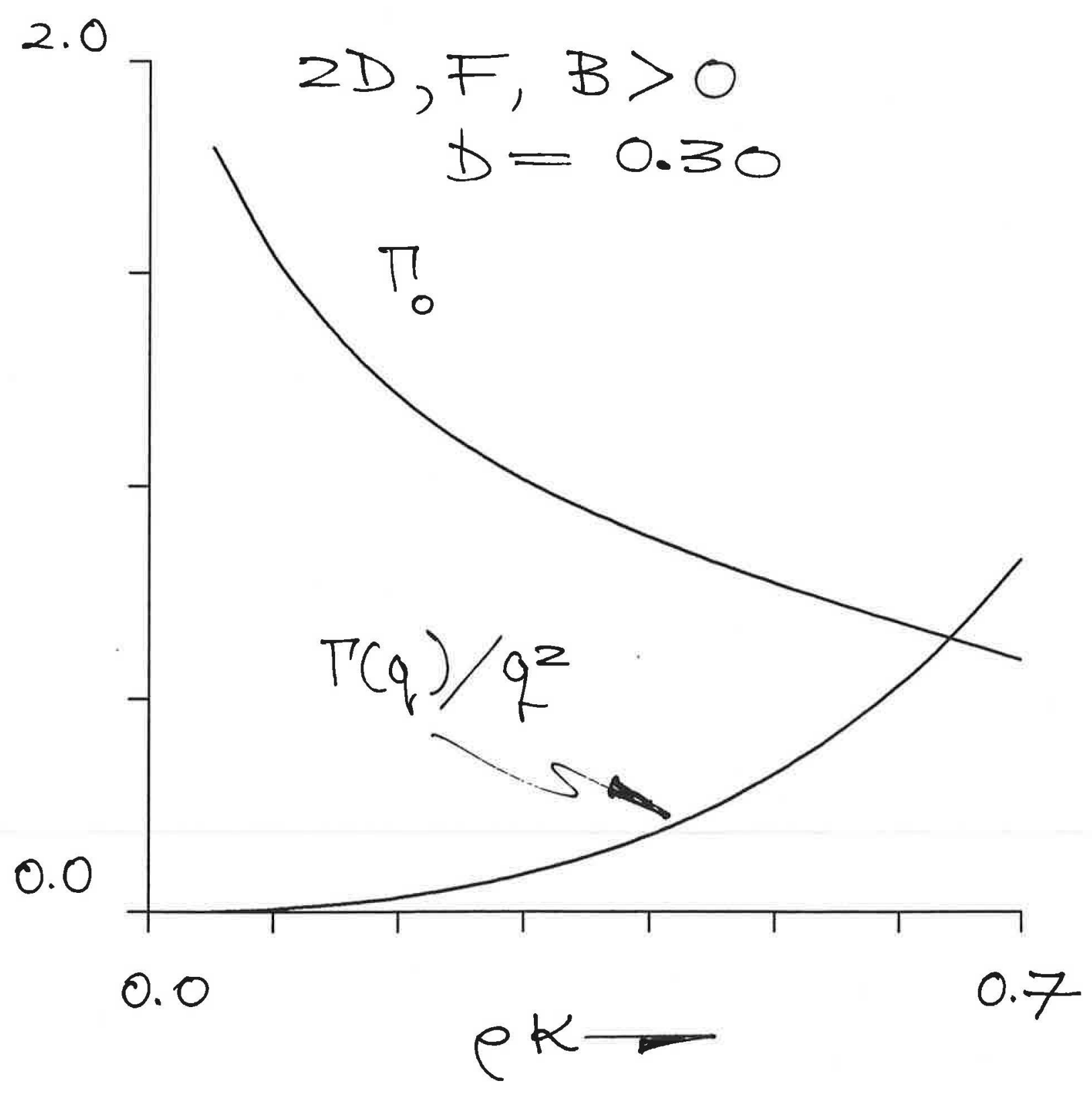

is 


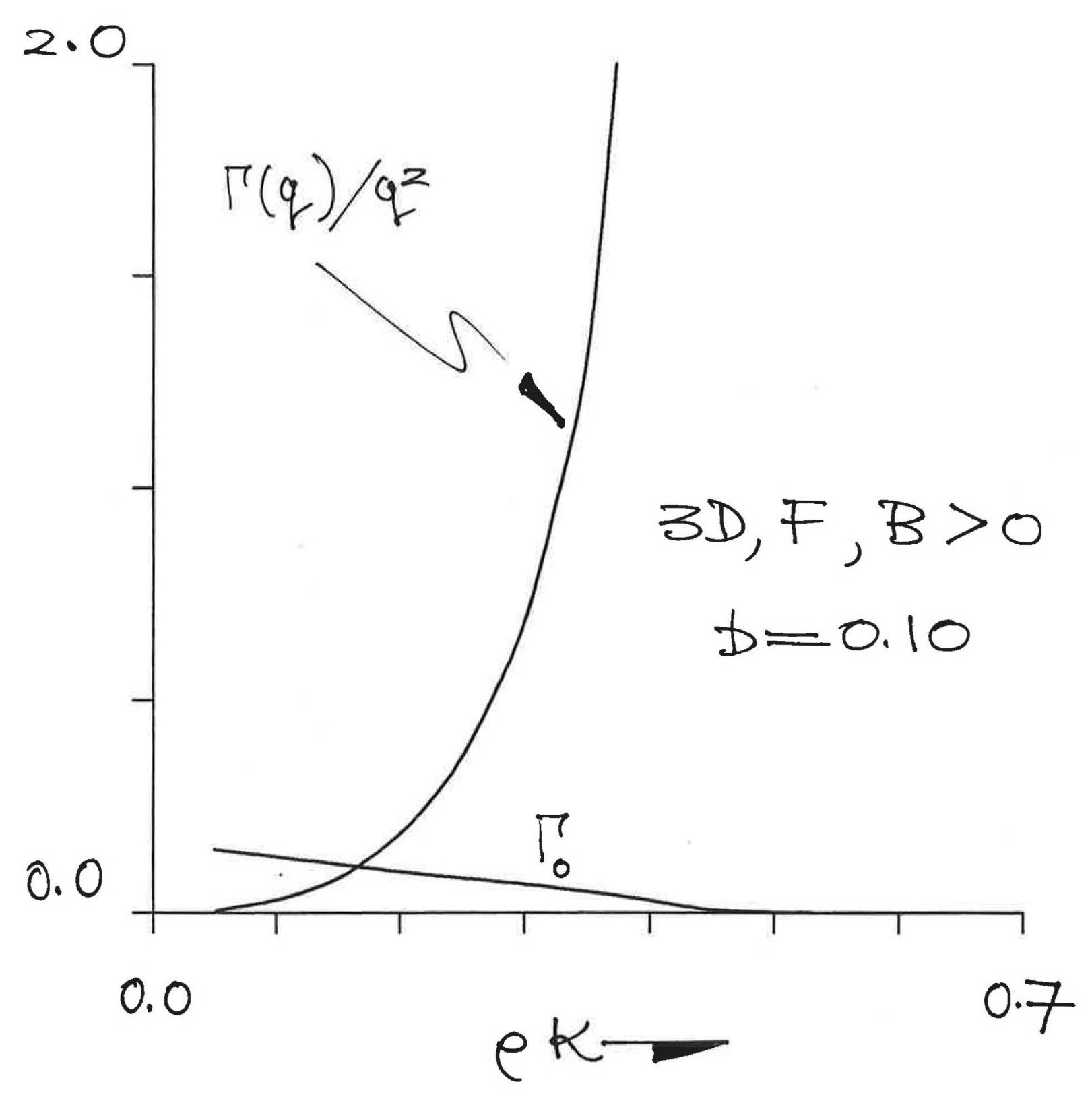

$2 a$ 


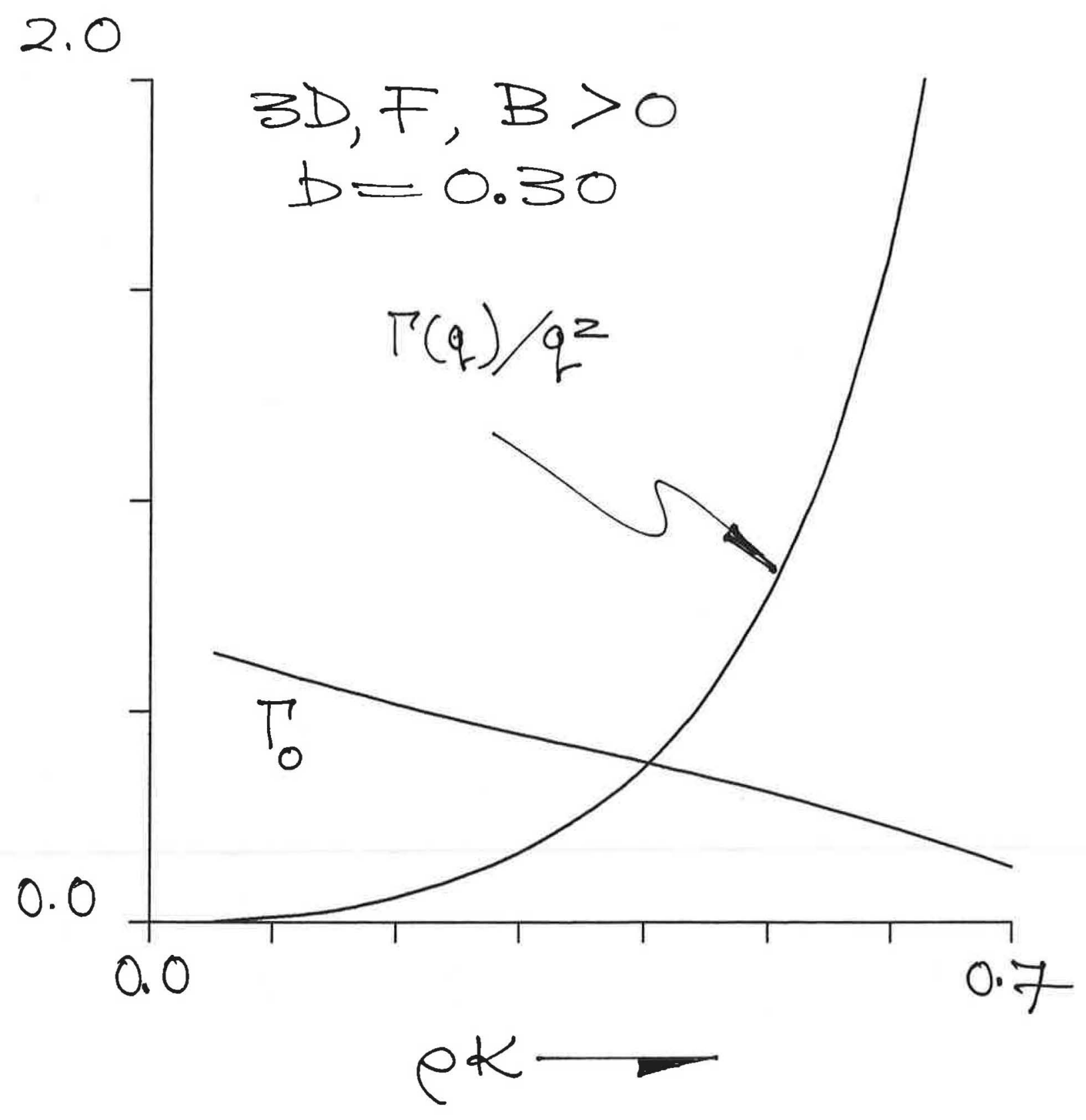

$2 b$ 

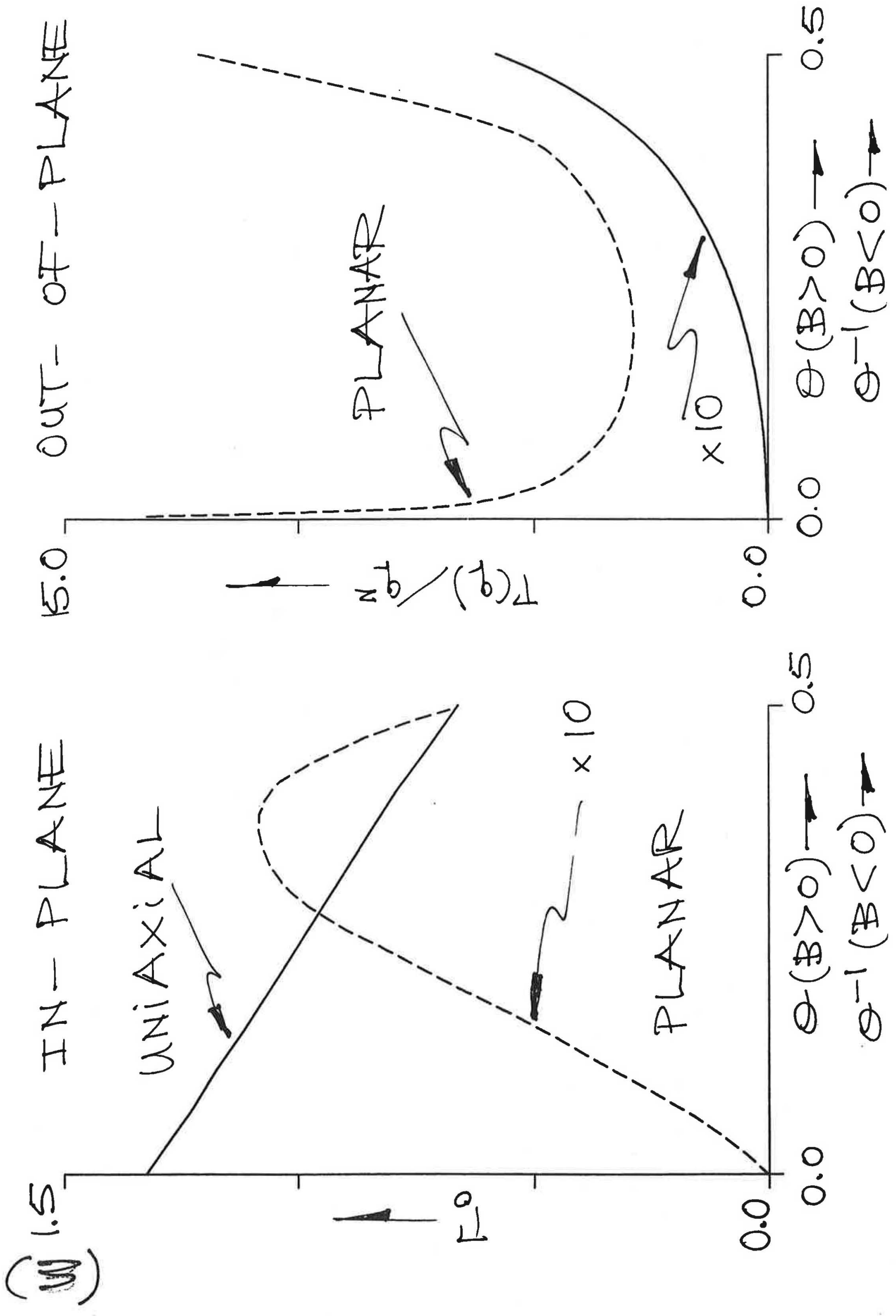


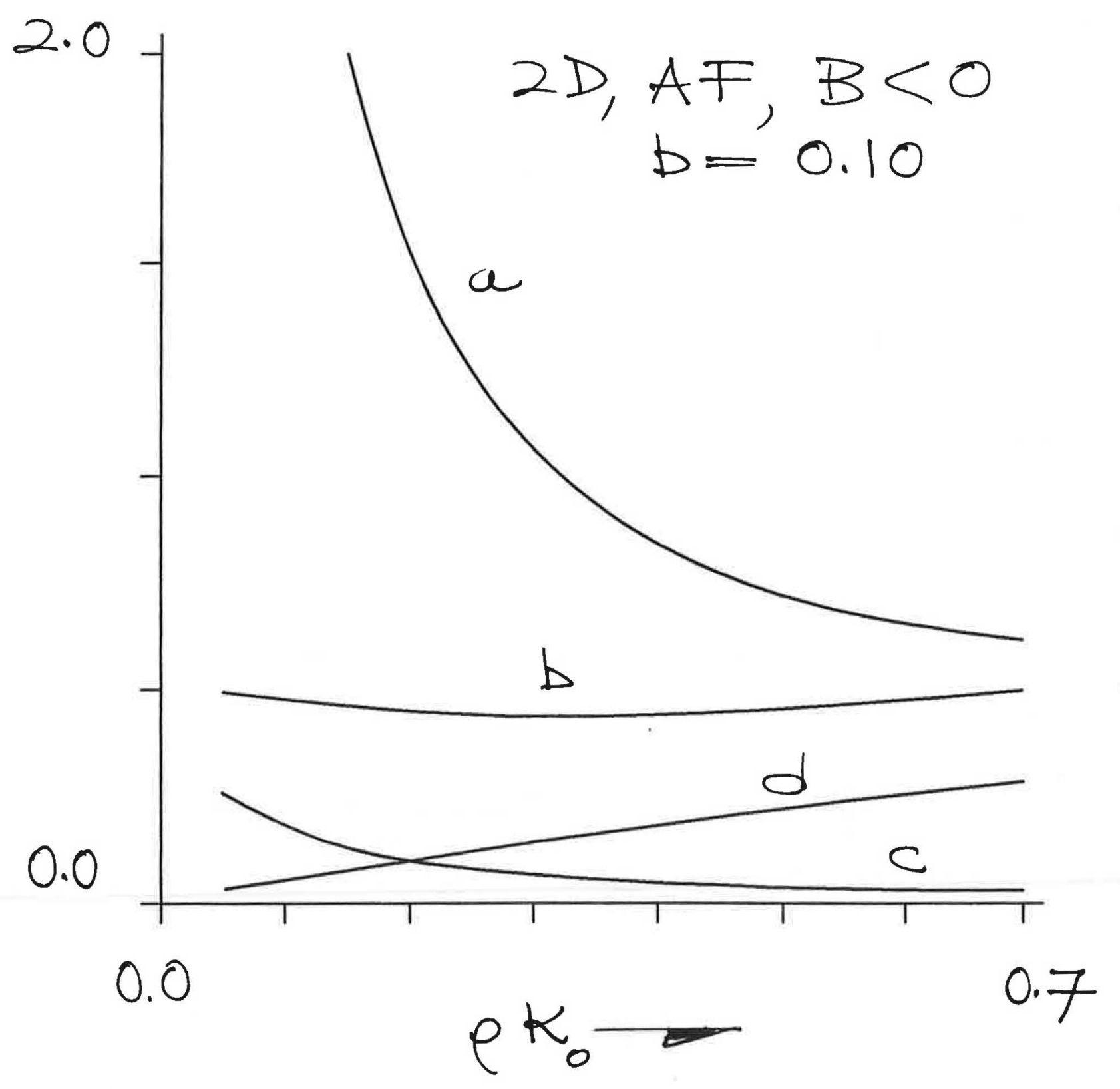

4 


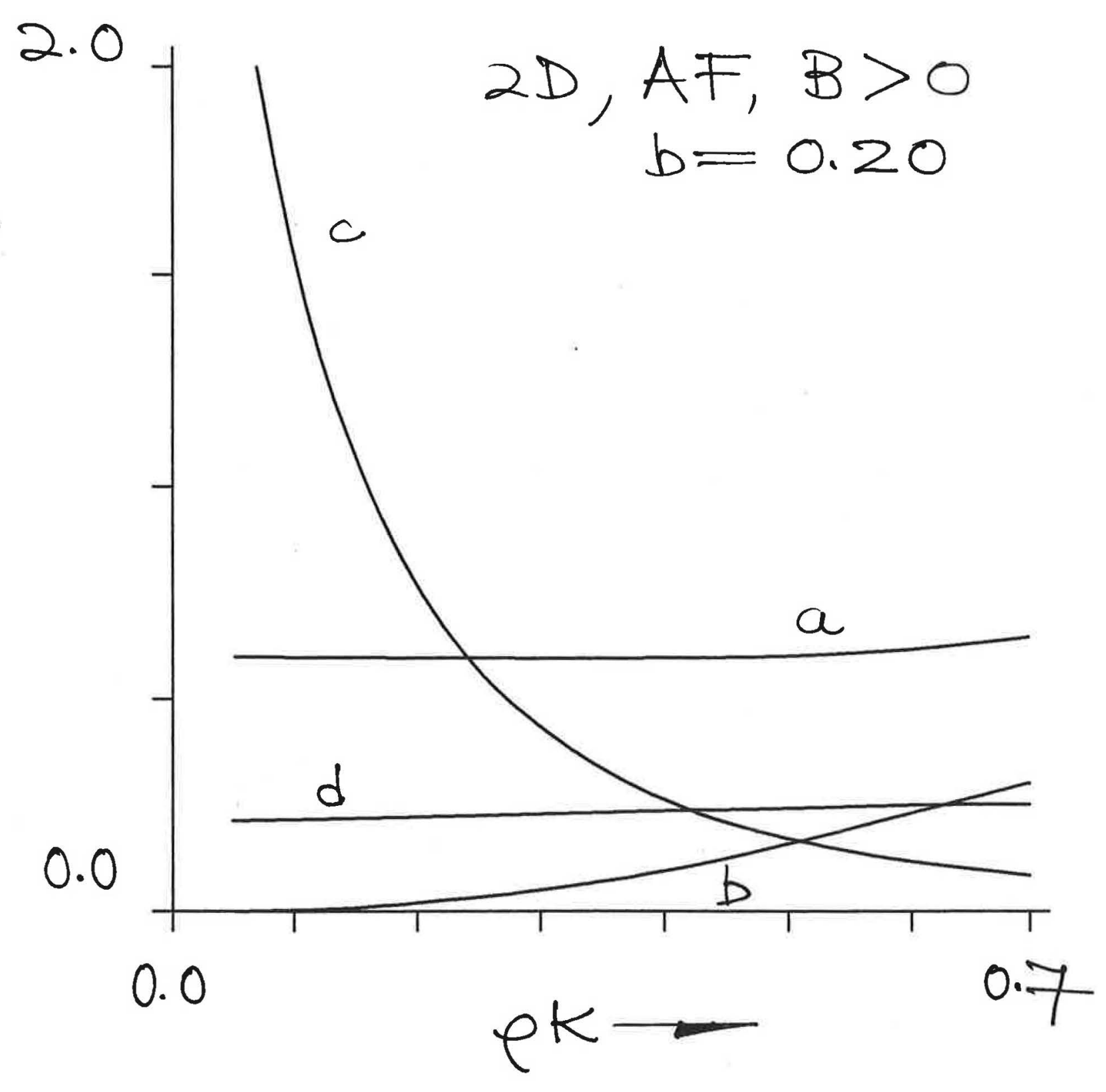

5 


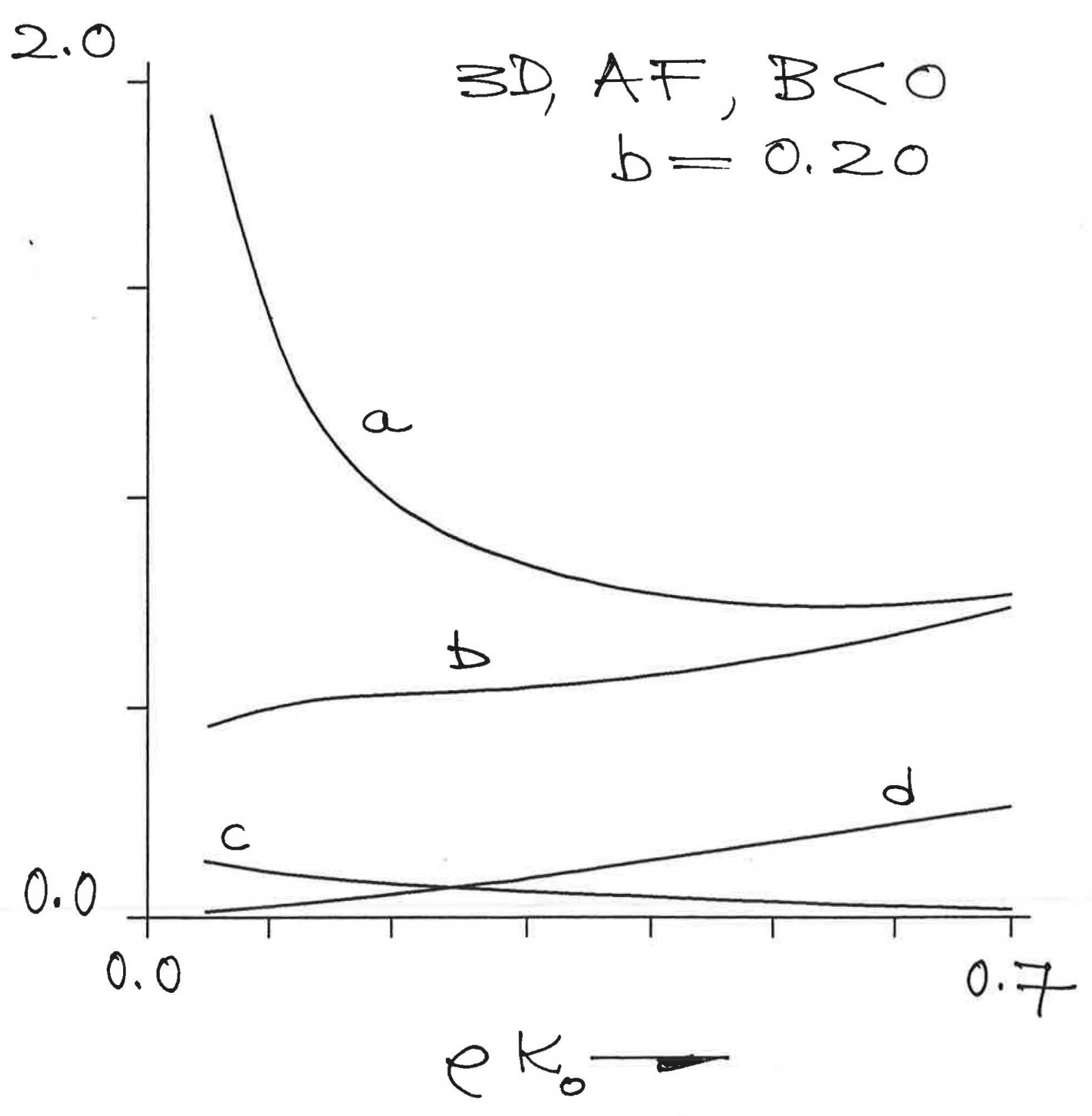

6 


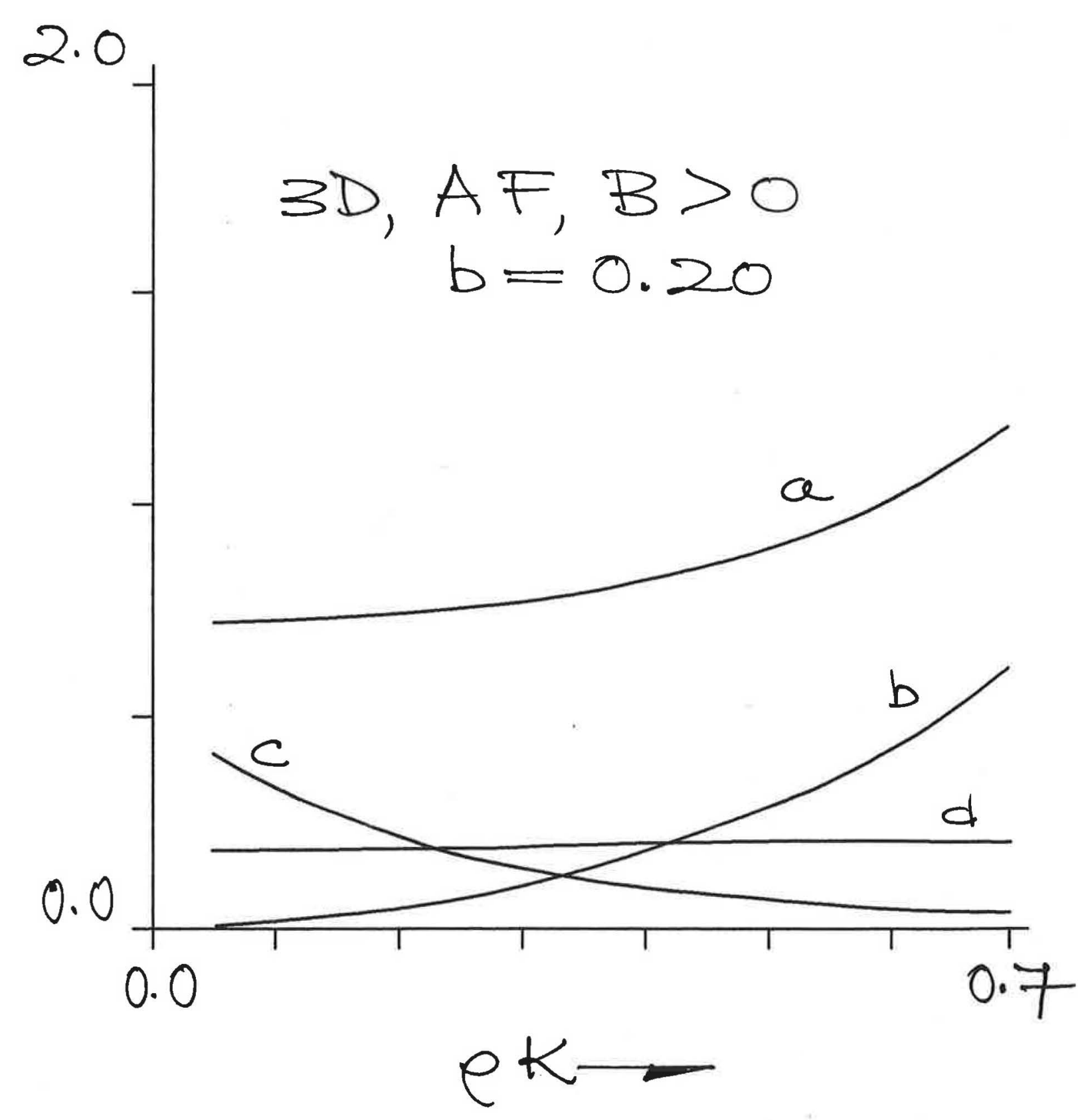

7 

